\title{
O tempo e o tipo de embalagem sobre a erva-mate tipo chimarrão durante armazenagem em condições ambientais
}

\author{
Influence of time and kind of packaging on mate during storage under ambient conditions
}

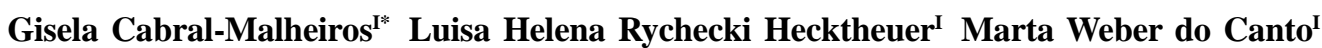 \\ Geisi Mello BalsamoI
}

\section{RESUMO}

O objetivo deste estudo foi avaliar a influência dos fatores embalagem e tempo na alteração da cor $e$ degradação da clorofila, durante armazenamento de ervamate tipo chimarrão em condições ambientais. Amostras de erva-mate, acondicionadas em embalagem de papel ou laminada, foram colocadas em câmara de armazenagem e analisadas em sete períodos, totalizando 12 semanas. Foram determinadas a concentração de clorofila ( $a, b$ e total), cor instrumental (CIELAB), umidade e atividade de água. Verificouse degradação dos pigmentos clorofílicos nas amostras em embalagem laminada (redução de 59,5\% na concentração de clorofila a e de $28,0 \%$ na de clorofila b) e resultados controversos para as amostras em embalagem de papel, os quais provavelmente tiveram interferência da reação de Maillard. As alterações de cor durante o período de armazenamento foram: redução no valor de $L^{*}$ para a ervamate acondicionada em embalagem de papel (de 49,3 para $48,1)$ e aumento de $L^{*}$ para o produto em embalagem laminada (de 49,3 para 51,5); redução de $-a^{*}$ e redução de $b^{*}$ para o produto de ambas as embalagens, papel ( $a^{*}=$ de 13,2 para $-5,9 ; b^{*}=$ de 29,3 para 28,5$)$ e laminada $\left(a^{*}=d e-\right.$ 13,3 para $-7,0 ; b^{*}=$ de 29,5 para 24,9). Concluiu-se que o tempo de armazenagem e o tipo de embalagem influenciaram significativamente $(P<0,05)$ os itens analisados, e a alteração de cor foi provavelmente influenciada pelas reações de feofitinização e oxidação dos pigmentos clorofílicos da ervamate.

Palavras-chave: erva-mate, embalagem, armazenamento, coloração.

\section{ABSTRACT}

The aim of this research was to evaluate the influence of packaging and time factors in color change and degradation of chlorophyll during storage of mate, mate type, in environmental conditions. Samples of mate, packaged in laminated flexible film or paper, were placed in a storage chamber, and analyzed at seven periods, during 12 weeks. Chlorophyll ( $a, b$ and total), instrumental color (CIELAB), moisture and water activity were determined. In the analysis of chlorophyllian pigments, it was verified their degradation in the samples packaged in laminated film (a reduction of $59.5 \%$ in chlorophyll $a$ and $28.0 \%$ in chlorophyll b), and controversial results were found in the samples on paper package. This result probably suffered the interference of Maillard reaction. The mate color changes during storage were: $L^{*}$ reduction in the samples on paper package (from 49.3 to 48.1 ) and $L^{*}$ increase in the samples on laminated package (from 49.3 to 51.5); - $a^{*}$ reduction and $b^{*}$ reduction in the product on both packages, paper ( $a^{*}=$ from -13.2 to $-5.9 ; b^{*}=$ from 29.3 to 28.5 ) and laminated film ( $a^{*}=$ from -13.3 to $-7.0 ; b^{*}=$ from 29.5 to 24.9 ). It was concluded that the storage time and the kind of package significantly influenced $(P<0.05)$ the items analized and that the color change was probably influenced by pheophytinization as well as by oxidation of chlorophyllian pigments in mate.

Key words: mate, package, storage, color.

\section{INTRODUÇÃO}

A Ilex paraguariensis A. St.-Hill., espécie conhecida popularmente como erva-mate, é nativa da América do Sul e tem sua área de ocorrência natural restrita, da qual cerca de $80 \%$ pertence ao Brasil. A região Sul do país é a maior produtora, além de consumidora, devido à tradição do chimarrão (CÂMARA, 2000; IBGE, 2007). A partir dos ramos da erva-mate obtém-se o produto homônimo, que tem

IDepartamento de Tecnologia e Ciência dos Alimentos, Centro de Ciências Rurais (CCR), Universidade Federal de Santa Maria (UFSM). Fx. de Camobi, km 9, Campus Universitário, 97105-900, Santa Maria, RS, Brasil. E-mail: cabralmalheiros@gmail.com. *Autor para correspondência. 
como índice de qualidade mais importante para os consumidores brasileiros a cor verde. Um dos maiores desafios do setor ervateiro é a instabilidade dessa cor verde-brilhante durante a armazenagem da erva-mate. $\mathrm{Na}$ tentativa de ofertar um produto com melhor qualidade, o industrial ervateiro tem utilizado diferentes tipos de embalagem e, gradualmente, tem diminuído a vida de prateleira da erva-mate, o que requer o recolhimento do produto disponibilizado na gôndola do supermercado, gerando um grande custo para o setor ervateiro (SANTOS, 2004).

A cor verde da erva-mate deve-se à presença de clorofila. A intensidade do verde tem relação direta com a concentração desse pigmento (MORAWICKI et al., 1999). Diversos fatores podem levar à degradação da clorofila, sendo sensível a alterações de $\mathrm{pH}$, enzimas, oxigênio, temperatura e luz, e a velocidade das reações é determinada pela atividade de água do alimento (LAJOLO et al., 1971; SCHWARTZ \& LORENZO, 1990; BOHN \& WALCZYK, 2004). Em vegetais desidratados, como a erva-mate, a feofitinização e a oxidação são as reações mais prováveis de degradação da clorofila, as quais produzem derivados com características espectrais diferentes do pigmento-mãe (LAJOLO \& LANFER-MARQUEZ, 1982; KING et al., 2001; SANTOS, 2004), alterando a cor do produto. Na reação de feofitinização, o magnésio do centro da molécula de clorofila é substituído por hidrogênio, de forma que a cor verde vívida da clorofila passa a uma cor marromazeitona, característica da feofitina. Devido ao fato de essa reação geralmente ter uma taxa de degradação mais elevada que outras vias de degradação da clorofila, ela é considerada o mecanismo mais importante de destruição da clorofila em alimentos processados (STREIT et al., 2005).

A avaliação instrumental de cor, pelo sistema CIELAB, tem sido utilizada na tentativa de medir essa perda de qualidade, a qual é subjetivamente percebida pelo consumidor. A redução do valor de $-\mathrm{a}^{*}$ já foi verificada em diversos estudos no processamento e na armazenagem da erva-mate (SCHMALKO \& ALZAMORA, 2001; SANTOS, 2004; SCHMALKO et al., 2005; VALDUGA et al., 2005), a qual tem sido atribuída à perda de clorofila e/ou da cor verde brilhante.

Nesse sentido, faz-se necessário um paralelo entre perda de cor e pigmento, considerando-se a absorção de umidade durante o armazenamento, nos dois tipos de embalagem mais utilizados atualmente pelas ervateiras no Brasil. Por meio dessa compreensão, é possível um melhor manejo por parte do setor ervateiro, a fim de minimizar prejuízos e proporcionar mais qualidade ao consumidor da erva-mate para chimarrão.
O objetivo deste estudo foi avaliar a influência do tipo de embalagem e do tempo durante armazenagem de erva-mate tipo chimarrão em condições ambientais.

\section{MATERIAL E MÉTODOS}

O produto erva-mate tipo chimarrão (Ilex paraguariensis A. St.-Hill.) foi fornecido por uma ervateira da região Noroeste do Rio Grande do Sul em unidades com capacidade de $1 \mathrm{~kg}$ cada e acondicionado nas embalagens de estudo. Esse produto constituiuse de uma mistura de matérias-primas de procedência variada: erva-mate nativa da região de Rio Negrinho, Santa Catarina (SC) - 44\%; erva-mate nativa da região de São Mateus do Sul, Paraná (PR) - 38\%; erva-mate cultivada da Argentina - 14\%; palito de erva-mate $4 \%$. As embalagens utilizadas comercialmente e no estudo foram: papel de dupla camada (papel couché e papel apergaminhado) e filme laminado (polipropileno biorientado metalizado/polietileno de baixa densidade). As amostras foram recebidas três dias após o processamento. No mesmo dia do recebimento, uma parte das amostras foi destinada para a armazenagem e outra para a realização imediata das análises físicoquímicas. Essa última fração da amostra foi considerada como tempo zero de armazenagem.

As amostras de erva-mate em embalagem laminada e em embalagem de papel foram distribuídas na câmara de armazenagem de forma homogênea e de uma só vez. De construção artesanal, a câmara de armazenagem pertence ao Núcleo de Pesquisa em Póscolheita (NPP) do Departamento de Fitotecnia da Universidade Federal de Santa Maria (UFSM). A cada duas semanas, seis unidades de erva-mate eram retiradas aleatoriamente, três de cada embalagem, para serem submetidas às análises físico-químicas. Ao todo, houve sete períodos de armazenagem: zero, duas, quatro, seis, oito, 10 e 12 semanas.

A umidade relativa (UR) foi mantida em $75 \%$ $( \pm 5 \%)$, e a temperatura média foi de $25^{\circ} \mathrm{C}\left( \pm 2,5^{\circ} \mathrm{C}\right)$. A temperatura e a umidade relativa escolhidas simulam condições ambientais médias de armazenagem e estão de acordo com o proposto por MAN \& JONES (1999). A incidência de luz no interior da câmara pode ser considerada desprezível.

As análises físico-químicas foram realizadas em triplicata, nos laboratórios do Departamento de Tecnologia e Ciência de Alimentos (DTCA) da UFSM, e iniciadas imediatamente após a retirada das amostras da câmara de armazenagem. Previamente à determinação de clorofila e à análise de cor, a erva-mate foi peneirada para a remoção dos palitos (fragmentos secos de ramos). 
A operação foi realizada manualmente, utilizando-se peneira de ensaio com abertura de malha de $800 \mu \mathrm{m}$.

A umidade foi determinada em estufa, a $105^{\circ} \mathrm{C}$ (INSTITUTO ADOLFO LUTZ, 1985), e a atividade de água foi obtida por um medidor de umidade Testo 650, seguindo-se os procedimentos de análise do fabricante.

Para a determinação de clorofila, primeiramente foi efetuada extração de pigmentos adaptando-se o método de YAMAUCHI et al. (1997) e utilizando-se como solvente N,N-dimetilformamida. A determinação espectrofotométrica da concentração de clorofila ( $a, b$ e total) da erva-mate foi efetuada conforme método proposto por MORAN (1982), obtendo-se a concentração dos pigmentos em $\mathrm{mg} \mathrm{g}^{-1}$ amostra (b.s.). Os comprimentos de onda utilizados foram 647, 654, 664 e 666nm, em espectrofotômetro UV/ Vis (Spectrum 22ED).

Os parâmetros de cor $L^{*}, a^{*}$ e $b^{*}$ foram obtidos por meio de um colorímetro tristimulus Minolta CR 310, operando no sistema CIELAB, em que L* corresponde à luminosidade e $a^{*}$ e $b^{*}$ são as coordenadas de cromaticidade $(-a=$ verde e $+\mathrm{a}=$ vermelho; $-\mathrm{b}=\mathrm{azul} \mathrm{e}+\mathrm{b}=$ amarelo). Previamente às leituras de cor, as amostras de erva-mate peneiradas foram acondicionadas e compactadas em placas de Petri. As leituras foram realizadas em triplicata, sob iluminante D65 e ângulo $10^{\circ}$.

O delineamento experimental utilizado foi o inteiramente casualizado (DIC), em esquema fatorial $2 \times 7$ (embalagem de papel e laminada $\mathrm{x}$ sete períodos de armazenagem), com três repetições. A unidade experimental foi $1 \mathrm{~kg}$ de erva-mate. Os dados foram submetidos à análise de variância (ANOVA), e o teste de Tukey foi utilizado para comparação de médias (ao nível de 5\% de significância). Para investigar possíveis relações entre as variáveis em estudo, realizou-se análise de correlação linear simples. A análise estatística foi realizada com o auxílio do software The SAS System for Windows versão 6.11 .

\section{RESULTADOS E DISCUSSÃO}

A tabela 1 mostra que houve absorção de umidade do ambiente e consequente aumento da atividade de água durante o período de armazenagem. A análise de variância (ANOVA) revela que ambas as variáveis foram significativamente $(\mathrm{P}<0,05)$ influenciadas pelo tempo de armazenagem e pela embalagem. A absorção de umidade das amostras em embalagem de papel foi significativamente maior que das amostras em embalagem laminada, conforme o teste de Tukey $(\mathrm{P}<0,05)$, e isso também ocorreu para a atividade de água. No produto acondicionado em embalagem laminada, não houve variação significativa de umidade no período de tempo estudado, e a atividade de água manteve-se praticamente estável. Por outro lado, no produto em embalagem de papel, houve grande variação nas primeiras quatro semanas, com posterior tendência à estabilização.

Tabela 1 - Variação média da umidade, atividade de água e concentração de clorofila $a$, clorofila $b$ e clorofila total em amostras de erva-mate armazenadas a $25^{\circ} \mathrm{C}\left( \pm 2,5^{\circ} \mathrm{C}\right)$ e $75 \%( \pm 5 \%)$ de UR por 12 semanas em embalagens comerciais. Santa Maria, 2006.

\begin{tabular}{|c|c|c|c|c|c|c|c|}
\hline Tratamentos & 0 & 2 & 4 & 6 & 8 & 10 & 12 \\
\hline & \multicolumn{7}{|c|}{ 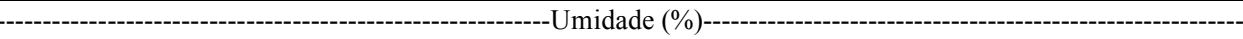 } \\
\hline Papel & $4,82^{\mathrm{d}}$ & $7,48^{\mathrm{c}}$ & $10,31^{\mathrm{b}}$ & $10,36^{\mathrm{b}}$ & $10,46^{\mathrm{b}}$ & $11,92^{\mathrm{a}}$ & $9,68^{\mathrm{b}}$ \\
\hline Laminado & $4,84^{d}$ & $4,90^{d}$ & $4,95^{\mathrm{d}}$ & $4,80^{\mathrm{d}}$ & $5,15^{\mathrm{d}}$ & $5,22^{\mathrm{d}}$ & $5,17^{\mathrm{d}}$ \\
\hline Papel & $0,41^{\mathrm{e}, \mathrm{f}}$ & $0,62^{d}$ & $0,71^{\mathrm{b}, \mathrm{c}}$ & $0,74^{\mathrm{a}, \mathrm{b}}$ & $0,71^{\mathrm{b}, \mathrm{c}}$ & $0,77^{\mathrm{a}}$ & $0,68^{\mathrm{c}}$ \\
\hline Laminado & $0,39^{\mathrm{f}}$ & $0,41^{\mathrm{e}, \mathrm{f}}$ & $0,40^{\mathrm{e}, \mathrm{f}}$ & $0,44^{\mathrm{e}}$ & $0,43^{\mathrm{e}, \mathrm{f}}$ & $0,44^{\mathrm{e}}$ & $0,44^{\mathrm{e}}$ \\
\hline Papel & $2,98^{\mathrm{a}}$ & $2,15^{\mathrm{c}, \mathrm{d}}$ & $1,89^{\mathrm{d}, \mathrm{e}}$ & $\begin{array}{c}a, \mathrm{mg} g \\
1,92^{\mathrm{d}, \mathrm{e}}\end{array}$ & $2,95^{\mathrm{a}}$ & $2,56^{\mathrm{b}}$ & $2,31^{\mathrm{b}, \mathrm{c}}$ \\
\hline Laminado & $2,94^{\mathrm{a}}$ & $2,15^{\mathrm{c}, \mathrm{d}}$ & $1,63^{e, f, g}$ & $1,57^{\mathrm{f}, \mathrm{g}}$ & $1,78^{\mathrm{e}, \mathrm{f}}$ & $1,46^{\mathrm{g}, \mathrm{h}}$ & $1,19^{\mathrm{h}}$ \\
\hline & & & ---------- & $\mathrm{la} b, \mathrm{mg} \mathrm{g}$ & ----------. & & \\
\hline Papel & $1,05^{\mathrm{a}}$ & $0,90^{\mathrm{b}}$ & $0,86^{\mathrm{b}, \mathrm{c}}$ & $0,84^{\mathrm{b}, \mathrm{c}}$ & $0,61^{\mathrm{d}}$ & $0,62^{d}$ & $0,63^{d}$ \\
\hline Laminado & $1,07^{\mathrm{a}}$ & $0,90^{\mathrm{b}}$ & $0,84^{\mathrm{b}, \mathrm{c}}$ & $0,88^{\mathrm{b}}$ & $0,84^{\mathrm{b}}$ & $0,76^{\mathrm{b}, \mathrm{c}}$ & $0,77^{\mathrm{c}}$ \\
\hline & & & -----C & total, $\mathrm{mg}$ & ) ------- & & \\
\hline Papel & $4,04^{\mathrm{a}}$ & $3,05^{\mathrm{c}, \mathrm{d}}$ & $2,74^{\mathrm{d}, \mathrm{e}, \mathrm{f}}$ & $2,75^{\mathrm{d}, \mathrm{e}, \mathrm{f}}$ & $3,56^{b}$ & $3,18^{\mathrm{c}}$ & $2,94^{\mathrm{c}, \mathrm{d}, \mathrm{e}}$ \\
\hline Laminado & $4,00^{\mathrm{a}}$ & $3,05^{\mathrm{c}, \mathrm{d}}$ & $2,47^{\mathrm{f}, \mathrm{g}}$ & $2,45^{f, g}$ & $2,62^{\mathrm{e}, \mathrm{f}}$ & $2,22^{\mathrm{g}, \mathrm{h}}$ & $1,96^{\mathrm{h}}$ \\
\hline
\end{tabular}

Os dados mostrados representam a média de três repetições. Letras diferentes correspondem a diferenças significativas entre as duas embalagens e os sete períodos de análise pelo teste de Tukey $(\mathrm{P}<0,05)$.

Ciência Rural, v.40, n.3, mar, 2010. 
A concentração média de clorofila total no tempo inicial para o lote de erva-mate foi de $4,02 \pm 0,02 \mathrm{mg}$ $\mathrm{g}^{-1}$ (b.s.). A literatura apresenta valores de 0,51 a $6,04 \mathrm{mg}$ $\mathrm{g}^{-1}$ (b.s.) para clorofila total em erva-mate no tempo inicial de armazenamento (MORAWICKI et al., 1999; SCHMALKO \& ALZAMORA, 2001; SANTOS, 2004), de forma que o valor obtido neste trabalho encontrase dentro desse intervalo. A concentração de clorofila no produto pode variar de acordo com a origem da matéria-prima (região, tipo de erval), o processamento, o método de quantificação e o tempo entre o processamento e a análise (SAAP, 1997; CÂMARA, 2000).

A literatura prevê redução exponencial da clorofila durante a armazenagem de alimentos (STEET \& TONG, 1996; MORAWICKI et al., 1999; SANTOS, 2004), o que foi observado nas amostras acondicionadas em embalagem de papel apenas na clorofila $b$. Obtiveram-se resultados inesperados para clorofila $a$ e total na embalagem de papel notadamente a partir da oitava semana, quando se observou um aumento significativo na concentração do pigmento (Tabela 1). Isso pode ter ocorrido por interferência de outros pigmentos formados durante a armazenagem da erva-mate, desde que apresentem absorção nos comprimentos de onda utilizados para o cálculo dos pigmentos clorofílicos, o que é permitido pelas limitações da espectrofotometria (BERSET \& CANIAUX, 1983).

Acredita-se que tenham sido reunidas condições ideais de substrato, atividade de água e tempo para o desencadeamento da reação de escurecimento não enzimático (ou reação de Maillard) na erva-mate armazenada em embalagem de papel. O pico da velocidade da reação de escurecimento não enzimático ocorre em atividades de água entre $0,55 \mathrm{e}$ 0,7 , típicas de vegetais desidratados (CHEFTEL \& CHEFTEL, 1992; JAYARAMAN \& DAS GUPTA, 1995). Conforme a tabela 1, esses valores estão mais próximos da atividade de água encontrada na erva-mate em embalagem de papel a partir de duas semanas de armazenagem. Já a atividade de água na erva-mate em embalagem laminada manteve-se abaixo desse intervalo por todo o período. Isso sugere que o comportamento inesperado do gráfico da concentração de clorofila possa ter sido causado pela interferência dos pigmentos formados na reação de Maillard.

$\mathrm{Na}$ erva-mate em embalagem laminada (Tabela 1), houve redução significativa para clorofila $a, b$ e total em função do tempo, principalmente nas quatro primeiras semanas de armazenagem. Em valores absolutos, houve uma queda de $59,5 \%$ na concentração de clorofila $a$ e de clorofila $b$ de apenas $28,0 \%$ ao final do período de armazenagem. Embora ambas sejam verdes, as clorofilas $a$ e $b$ têm espectros de absorção ligeiramente diferentes, de maneira que para o olho humano a clorofila $a$ apresenta uma tonalidade verdeazulada, e a clorofila $b$, verde-amarelada (STREIT et al., 2005). A alteração na proporção entre as duas formas da clorofila pode estar relacionada à alteração da cor no produto erva-mate durante a armazenagem.

Os valores de $\mathrm{L}^{*}, \mathrm{a}^{*} \mathrm{e} \mathrm{b}^{*}$ obtidos pela análise instrumental de cor são mostrados na tabela 2. A análise de variância identificou influência significativa da embalagem e do tempo sobre as três coordenadas do espaço de cores CIELAB. Observa-se que os valores encontrados para o parâmetro a* foram negativos, que corresponde à cor verde, e do parâmetro $b^{*}$ positivos (amarelo) em todas as análises realizadas.

Tabela 2 - Variação média dos valores de $\mathrm{L}^{*}$, a* e $\mathrm{b}^{*}$ em amostras de erva-mate armazenadas a $25^{\circ} \mathrm{C}\left( \pm 2,5^{\circ} \mathrm{C}\right)$ e $75 \%( \pm 5 \%)$ de $\mathrm{UR}$ por 12 semanas em embalagens comerciais. Santa Maria, 2006.

\begin{tabular}{|c|c|c|c|c|c|c|c|}
\hline \multirow{2}{*}{ Tratamentos } & \multicolumn{7}{|c|}{-Tempo de armazenamento (semanas)-- } \\
\hline & 0 & 2 & 4 & 6 & 8 & 10 & 12 \\
\hline & \multicolumn{7}{|c|}{ 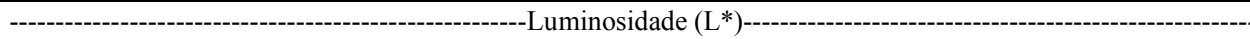 } \\
\hline Papel & $49,26^{\mathrm{c}}$ & $48,09^{\mathrm{d}, \mathrm{e}}$ & $47,60^{\mathrm{e}}$ & $47,86^{\mathrm{e}}$ & $48,04^{\mathrm{d}, \mathrm{e}}$ & $47,51^{\mathrm{e}}$ & $48,14^{\mathrm{d}, \mathrm{e}}$ \\
\hline Laminado & $49,28^{\mathrm{c}}$ & $48,72^{\mathrm{c}, \mathrm{d}}$ & $49,01^{\mathrm{c}}$ & $49,50^{\mathrm{c}}$ & $50,45^{b}$ & $51,32^{\mathrm{a}}$ & $51,47^{\mathrm{a}}$ \\
\hline Papel & $-13,20^{i}$ & $-11,98^{h}$ & $-10,51^{\mathrm{f}}$ & $\begin{array}{l}\text { metro de } \\
-8,91^{d}\end{array}$ & $-7,55^{c}$ & $-6,60^{b}$ & $-5,90^{a}$ \\
\hline \multirow[t]{2}{*}{ Laminado } & $-13,27^{i}$ & $-12,50^{h}$ & $-11,06^{\mathrm{g}}$ & $-9,82^{e}$ & $-8,70^{d}$ & $-7,87^{\mathrm{c}}$ & $-7,02^{b}$ \\
\hline & & & ---------- & netro de c & ------------ & & \\
\hline Papel & $29,33^{\mathrm{a}, \mathrm{b}}$ & $28,69^{\mathrm{c}}$ & $28,86^{\mathrm{c}}$ & $28,87^{\mathrm{c}}$ & $28,88^{\mathrm{b}, \mathrm{c}}$ & $28,64^{\mathrm{c}, \mathrm{d}}$ & $28,46^{\mathrm{c}, \mathrm{d}}$ \\
\hline Laminado & $29,54^{\mathrm{a}}$ & $28,86^{\mathrm{c}}$ & $28,20^{\mathrm{d}, \mathrm{e}}$ & $27,81^{\mathrm{e}}$ & $27,06^{\mathrm{f}}$ & $26,32^{\mathrm{g}}$ & $24,88^{\mathrm{h}}$ \\
\hline
\end{tabular}

Os dados mostrados representam a média de três repetições. Letras diferentes correspondem a diferenças significativas entre as duas embalagens e os sete períodos de análise pelo teste de Tukey $(\mathrm{P}<0,05)$. 
Por meio do teste de Tukey $(\mathrm{P}<0,05)$, observa-se que $\mathrm{L}^{*}$ elevou-se significativamente nas amostras em embalagem laminada ao longo do tempo de armazenagem, enquanto para a embalagem de papel houve uma pequena redução, também significativa, no início da armazenagem, mantendo-se constante no seu decorrer. Dessa forma, desde a quarta semana de armazenagem o valor de L* foi significativamente maior para o produto acondicionado em embalagem laminada que para o produto acondicionado na embalagem de papel. Para os parâmetros $a^{*}$ e $b^{*}$, o efeito do fator tempo foi de redução, de forma que para ambos os produtos (embalagens laminada e papel) houve decréscimo significativo nos valores absolutos de $\mathrm{a}^{*}$ e b* ao longo da armazenagem.

A luminosidade é uma coordenada do espaço de cores CIELAB que pode variar do 0 ao 100 , ou seja, do preto ao branco (LAWLESS \& HEYMANN, 1999). Sendo assim, pelos resultados obtidos, deduzse que a cor das amostras acondicionadas em embalagem laminada ficou mais clara e das amostras em embalagem de papel se tornou mais escura que a amostra no tempo zero.

O escurecimento das amostras em embalagem de papel pode estar relacionado com a reação de escurecimento não enzimático, hipótese levantada anteriormente na discussão dos pigmentos clorofílicos. Um decréscimo significativo do valor de L* foi observado por MORALES \& JIMÉNEZ-PÉREZ (2001) durante a formação de pigmentos na reação de Maillard, os quais ocasionaram escurecimento do meio reativo.

A comparação de médias pelo teste de Tukey mostrou diferença significativa do valor de $-\mathrm{a}^{*}$ entre as embalagens a partir da quarta semana de armazenagem, quando a erva-mate embalada em papel apresentou valores mais baixos. Para valores negativos do parâmetro a*, a aproximação do zero no espaço de cores corresponde à perda de cor verde (LAWLESS \& HEYMANN, 1999), a qual foi verificada para as amostras das duas embalagens, porém em maior intensidade para o produto acondicionado em embalagem de papel.

Foi realizada análise de correlação sobre os dados obtidos com a erva-mate em embalagem laminada, cruzando a concentração de clorofila com o parâmetro de cor $\mathrm{a}^{*}$. Optou-se por não realizar a mesma análise para a embalagem de papel, em razão da provável interferência de outros pigmentos, e por não se conhecer a extensão desta.

Houve correlação significativa $(\mathrm{P}<0,01)$ com clorofila $a(\mathrm{r}=0,86), b(\mathrm{r}=0,83)$ e total $(\mathrm{r}=0,87)$, o que relaciona a aproximação de zero na coordenada -a* com a diminuição na concentração de clorofila, fato observado também por outros autores (SCHMALKO \& ALZAMORA, 2001; SINNECKER et al., 2002; CABRAL-MALHEIROS et al., 2006). Sugere-se que essa correlação esteja ligada à reação de feofitinização da clorofila, o que vai de acordo com alguns trabalhos que relatam correlação entre a formação de feofitina e a diminuição no valor de -a* (STEET \& TONG, 1996; WEEMAES et al., 1999; KOCA et al., 2007). A conversão de clorofila em feofitina é o mecanismo mais comum de degradação da clorofila, tendo em vista que o $\mathrm{pH}$ da maioria dos vegetais favorece essa reação, e o fato de que, mesmo em valores de atividade de água muito baixos, ela pode ocorrer (LAJOLO \& LANFERMARQUEZ, 1982).

O parâmetro $b^{*}$, nas amostras em embalagem de papel, mostrou redução significativa apenas entre o tempo zero e as duas semanas de armazenagem, mantendo-se nos mesmos níveis no restante do período $(\mathrm{P}<0,05)$. Diferentemente, a erva-mate em embalagem laminada mostrou queda constante do valor de $b^{*}$ durante todo o período de estudo $(\mathrm{P}<0,05)$, conforme tabela 2. O decréscimo do valor positivo de $b^{*}$ no espaço de cores indica perda de amarelo (LAWLESS \& HEYMANN, 1999).

A análise de correlação entre o parâmetro de cor $b^{*}$ e os pigmentos clorofílicos, assim como ocorreu com o parâmetro $\mathrm{a}^{*}$ (considerando somente a embalagem laminada), também apresentou correlação significativa de $\mathrm{b}^{*}$ com clorofila $a, b$ e total $(\mathrm{r}=0,84$; $\mathrm{r}=0,82$ e $\mathrm{r}=0,85$, respectivamente).

$\mathrm{Na}$ literatura, vários autores têm avaliado a correlação do parâmetro b* com a concentração de clorofila. Em estudos com erva-mate, SCHMALKO et al. (2005) verificaram que a perda de clorofila não alterou o parâmetro b*; por outro lado, SCHMALKO \& ALZAMORA (2001) relataram correlação negativa entre o valor de $b^{*}$ e a concentração de clorofila. IHL et al. (1998), em estudo com alcachofra, verificaram redução de $b^{*}$ concomitante com a redução de clorofila (correlação positiva), assim como no presente estudo. Infere-se que a redução no valor de $b^{*}$ esteja relacionada à perda de clorofila, porém por outras vias, não pela feofitinização. WEEMAES et al. (1999), ao estudarem suco de brócolis, verificaram que a feofitinização não afetou o valor de $b^{*}$, sugerindo que a variação desse parâmetro ocorra por outros mecanismos de degradação da clorofila ou de outros compostos presentes na amostra. A faixa de atividade de água verificada na erva-mate em embalagem laminada favorece reações de oxidação (CHEFTEL \& CHEFTEL, 1992), o que poderia ter ocasionado degradação de pigmentos clorofílicos por mecanismos oxidativos. 
Considerando-se que a diminuição (aproximação do zero) no valor de $-\mathrm{a}^{*}$, no espaço de cores CIELAB, corresponde à perda de verde e que a diminuição no valor de $b^{*}$ corresponde à perda de amarelo (LAWLESS \& HEYMANN, 1999), pode-se estimar a cor final da erva-mate em estudo. Nas amostras em embalagem de papel, houve uma tendência predominante de aproximação do zero no eixo -a* e pequena redução de $b^{*}$, resultando num verde menos intenso, com o amarelo mais proeminente que a amostra inicial. Para as amostras em embalagem laminada, houve também redução no valor de $-\mathrm{a}^{*}$, porém queda acentuada do $b^{*}$, o que resulta em uma cor menos intensa que a inicial no verde e no amarelo, contudo menos amarelada que a erva-mate da embalagem de papel.

\section{CONCLUSÕES}

Após 12 semanas de armazenagem da ervamate tipo chimarrão em condições ambientais, em embalagem laminada ou papel, concluiu-se que houve degradação de clorofila $a$ e $b$, e alteração da cor (expressa nas coordenadas $\mathrm{L}^{*}, \mathrm{a}^{*} \mathrm{e} \mathrm{b}^{*}$ ), com influência significativa do tempo e da embalagem. Uma das principais diferenças entre as embalagens residiu na absorção de umidade, tendo em vista o incremento significativo de umidade e a atividade de água nas amostras com embalagem de papel.

\section{REFERÊNCIAS}

BERSET, C.; CANIAUX, P. Relationship between color evaluation and chlorophyllian pigment content in dried parsley leaves. Journal of Food Science, v.48, p.1854-1857, 1983. Disponível em: <http://dx.doi.org/10.1111/j.1365-2621.1983.tb05100.x>. Acesso em: 12 nov. 2009. doi: 10.1111/j.13652621.1983.tb05100.x.http://www3.interscience.wiley.com/ doiinfo.html.

BOHN, T.; WALCZYK, T. Determination of chlorophyll in plant samples by liquid chromatography using zinc-phthalocyanine as an internal standard. Journal of Chromatography A, v.1024, n.1-2, p.123-128, 2004. Disponível em: <http://dx.doi.org/ 10.1016/j.chroma.2003.10.067>. Acesso em: 12 nov. 2009. doi: 10.1016/j.chroma.2003.10.067.

CABRAL-MALHEIROS, G. et al. Comparação de métodos espectrofotométricos para determinação de clorofila e feofitina em erva-mate. In: CONGRESSO BRASILEIRO DE CIÊNCIA E TECNOLOGIA DE ALIMENTOS, 20., 2006, Curitiba, PR. Anais... Curitiba: SBCTA, 2006. 1 CD-ROM.

CÂMARA SETORIAL DA CADEIA PRODUTIVA DA ERVAMATE DO PARANÁ. Produtos alternativos e desenvolvimento da tecnologia industrial na cadeia produtiva da erva-mate. Curitiba, 2000. Série PADCT III, n. 1 .
CHEFTEL, J.C.; CHEFTEL, H. Introducción a la bioquímica y tecnología de los alimentos. Zaragoza: Acribia, 1992. 1v.

IHL, M. et al. Chlorophyllase inactivation as a measure of blanching efficacy and colour retention of artichokes (Cynara scolymus L.). LWT, v.31, n.1, p.50-56, 1998. Disponível em: $<$ http://dx.doi.org/10.1006/fstl.1997.0296>. Acesso em: 17 nov. 2009. doi: 10.1006/fstl.1997.0296.

IBGE. INSTITUTO BRASILEIRO DE GEOGRAFIA E ESTATÍSTICA. Produção da extração vegetal e da silvicultura 2007. Rio de Janeiro: IBGE, 2007. 47p.

INSTITUTO ADOLFO LUTZ. Normas Analíticas do Instituto Adolfo Lutz: métodos físicos e químicos para alimentos. 3.ed. São Paulo: IAL, 1985. 1v.

JAYARAMAN, K.S.; DAS GUPTA, D.K. Drying of fruits and vegetables. In: MUJUMDAR, A.S. Handbook of industrial drying. New York: Marcel Dekker, 1995. 1v. Cap.21, p.643690.

KING, V.A.E. et al. Chlorophyll stability in spinach dehydrated by freeze-drying and controlled low-temperature vacuum dehydration. Food Research International, v.34, n.2-3, p.167-175, 2001. Disponível em: <http://dx.doi.org/10.1016/ S0963-9969(00)00148-4>. Acesso em: 17 nov. 2009. doi: 10.1016/S0963-9969(00)00148-4.

KOCA, N. et al. Effect of $\mathrm{pH}$ on chlorophyll degradation and colour loss in blanched green peas. Food Chemistry, v.100, n.2, p.609-615, 2007. Disponível em: <http://dx.doi.org/ 10.1016/j.foodchem.2005.09.079>. Acesso em: 17 nov. 2009. doi: $10.1016 /$ j.foodchem.2005.09.079.

LAJOLO, F.M.; LANFER MARQUEZ, U.M. Chlorophyll degradation in a spinach system at low and intermediate water activities. Journal of Food Science, v.47, p.1995-1998, 1982. Disponível em: <http://dx.doi.org/10.1111/j.13652621.1982.tb12929.x>. Acesso em: 18 nov. 2009. doi: 10.1111/j.1365-2621.1982.tb12929.x

LAJOLO, F.M. et al. Reaction at limited water concentration. 2. Chlorophyll degradation. Journal of Food Science, v.36, p.850-853, 1971. Disponível em: <http://dx.doi.org/10.1111/ j.1365-2621.1971.tb15542.x>. Acesso em: 18 nov. 2009. doi: 10.1111/j.1365-2621.1971.tb15542.x.

LAWLESS, H.T.; HEYMANN, H. Sensory evaluation of food. Maryland: Aspen Publishers, 1999. 848p.

MAN, C.M.D.; JONES, A.A. Shelf life evaluation of foods. Gaithersburg: Aspen Publishers, 1999. 321p.

MORALES, F.J.; JIMÉNEZ-PÉREZ, S. Free radical scavenging capacity of Maillard reaction products as related to colour and fluorescence. Food Chemistry, v.72, n.1, p.119-125, 2001. Disponível em: <http:/ /dx.doi.org/10.1016/S0308-8146(00)00239-9>. Acesso em: 18 nov. 2009. doi: 10.1016/S0308-8146(00)00239-9.

MORAN, R. Formulae for determination of chlorophyllous pigments extracted with N,N-dimethylformamide. Plant Physiology, v.69, n.6, p.1376-1381, 1982. Disponível em: $<\mathrm{http}$ ://dx.doi.org/10.1104/pp.69.6.1376>. Acesso em: 18 nov. 2009. doi: $10.1104 /$ pp.69.6.1376. 
MORAWICKI, R.O. et al. Chlorophyll stability in yerba maté leaves in controlled atmospheres. Brazilian Archives of Biology and Technology, v.42, n.1, p.85-90, 1999.

SAAP. SECRETARIA DA AGRICULTURA E ABASTECIMENTO DO PARANÁ. Erva-mate: prospecção tecnológica da cadeia produtiva. Curitiba : SEAB, 1997. $130 \mathrm{p}$.

SANTOS, K.A. Estabilidade da erva-mate (Ilex paraguariensis St. Hill.) em embalagens plásticas. 2004. 109f. Dissertação (Mestrado em Tecnologia de Alimentos) Setor de Tecnologia, Universidade Federal do Paraná, Curitiba, PR.

SCHMALKO, M.E; ALZAMORA, S.M. Color, chlorophyll, caffeine, and water content variation during yerba-mate processing. Drying Technology, v.19, n.3, p.597-608, 2001. Disponível em: < http://dx.doi.org/10.1081/DRT-100103937>. Acesso em: 18 nov. 2009. doi: 10.1081/DRT-100103937.

SCHMALKO, M.E. et al. Effect of water activity and temperature in color and chlorophylls changes in yerba mate leaves. International Journal of Food Properties, v. 8 , n.2, p.313-322, 2005. Disponível em: <http://dx.doi.org/ 10.1081/JFP-200060250>. Acesso em: 18 nov. 2009. doi: $10.1081 /$ JFP-200060250.

SCHWARTZ, S.J.; LORENZO, T.V. Chlorophylls in foods. Critical Reviews in Food Science and Nutrition, v.29, n.1, p.1-17, 1990. Disponível em: <http://dx.doi.org/10.1080/ 10408399009527511>. Acesso em: 18 nov. 2009 . doi: $10.1080 / 10408399009527511$.

SINNECKER, P. et al. Relationship between color (instrumental and visual) and chlorophyll contents in soybean seeds during ripening. Journal of Agricultural and Food Chemistry, v.50, n.14, p.3961-3966, 2002. Disponível em: <http:// dx.doi.org/10.1021/jf0113023>. Acesso em: 18 nov. 2009. doi: $10.1021 / \mathrm{jf0} 113023$.

STEET, J.A.; TONG, C.H. Degradation kinetics of green color and chlorophylls in peas by colorimetry and HPLC. Journal of Food Science, v.61, n.5, p.924-931, 1996. Disponível em: $<$ http://dx.doi.org/10.1111/j.1365-2621.1996.tb10903.x>. Acesso em: 18 nov. 2009. doi: 10.1111/j.13652621.1996.tb10903.x.

STREIT, N.M. et al. As clorofilas. Ciência Rural, v.35, n.3, p.748-755, 2005. Disponível em: <http://dx.doi.org/10.1590/ S0103-84782005000300043>. Acesso em: 18 nov. 2009. doi: $10.1590 / \mathrm{S} 0103-84782005000300043$.

VALDUGA, E. et al. Nota prévia - Avaliação das características de qualidade da erva-mate (chimarrão) acondicionada em diferentes embalagens. Brazilian Journal of Food Technology, v.8, n.2, p.99-105, 2005.

WEEMAES, C.A. et al. Kinetics of chlorophyll degradation and color loss in heated broccoli juice. Journal of Agriculture and Food Chemistry, v.47, n.6, p.2404-2409, 1999. Disponível em: <http://dx.doi.org/10.1021/jf980663o>. Acesso em: 18 nov. 2009 . doi: $10.1021 /$ jf980663o.

YAMAUCHI, N. et al. In vitro chlorophyll degradation in stored brocolli (Brassica oleracea L. var. italica) florets. Postharvest Biology and Technology, v.12, n.3, p.239-245, 1997. Disponível em: <http://dx.doi.org/10.1016/S09255214(97)00063-X>. Acesso em: 18 nov. 2009. doi: 10.1016/ S0925-5214(97)00063-X. 